\title{
Production scheduling problem and solver improvement via integration of the grey wolf optimizer into the augmented Lagrangian relaxation method
}

\author{
Kamyar Tolouei $^{1} \cdot$ Ehsan Moosavi $^{1}$
}

Received: 6 June 2020 / Accepted: 23 October 2020 / Published online: 9 November 2020

(c) Springer Nature Switzerland AG 2020

\begin{abstract}
In open-pit mines, the long-term production scheduling problem is a mixed-integer programming optimization problem. Usually, it is tricky to reach the true optimal solution to the long-term production scheduling problem due to its complexity and dimensions. However, owing to its high-dimensional and combinatorial nature, the long-term production scheduling problem hinders the development of a precise mathematical optimization technique that can solve the whole problem for any real-size block model. In this paper, a hybrid model is introduced to solve the long-term production scheduling problem, taking into account grade uncertainty assisted by the augmented Lagrangian relaxation and the grey wolf optimizer methods. The hybrid augmented Lagrangian relaxation-the grey wolf optimizer technique is recommended to solve the long-term production scheduling problem to improve its performance and, consequently, speed up the convergence. The proposed model has been compared with the results of the hybrid methods gained from the classic Lagrangian relaxation and augmented Lagrangian relaxation methods integrated with the bat algorithm, particle swarm optimization, genetic algorithm, the traditional subgradient method, and conventional method without using the Lagrangian approach. The fallouts point out that better presentation is gained by the augmented Lagrangian relaxation-the grey wolf optimizer method in terms of net present value, average ore grade, and CPU time. Moreover, the cumulative net present value by the proposed model is $13.39 \%$ more than the conventional method.
\end{abstract}

Keywords Production scheduling · Optimization - Metaheuristic algorithms · Lagrangian relaxation · Open-pit mine · Grade uncertainty

\section{Introduction}

In open-pit mines, the long-term production scheduling (LTPS) is known as a complex optimization problem that is composed of input data uncertainty, large datasets, and various constraints. Uncertainties in mining projects are arising from inadequate and imperfect data. Different geological, economic, or technical factors subject to uncertainty in the input. The uncertainty caused by geological features is deliberated to be the most significant source of uncertainty among these factors as it is generally termed grade uncertainty. Geostatistical conditional simulation techniques develop a framework to measure grade uncertainty through generating multiple, equally probable simulated recognitions of the orebody. Over the last few years, a large number of models have been proposed to incorporate this grade uncertainty into the optimization process. However, it is generally complicated and

Ehsan Moosavi, se.moosavi@yahoo.com; se_moosavi@azad.ac.ir| 'Department of Petroleum and Mining Engineering, South Tehran Branch, Islamic Azad University, Tehran, Iran. 
computationally costly to solve these models for actualsized open-pit mines. Over the past few decades, researchers have greatly succeeded in this respect, as shown in Table 1. Accordingly, there has been a more comprehensive discussion on the allocation of optimal algorithms and the future of production planning. Despite the efforts of researchers, it is not possible to consider LTPS problem as a well-solved problem. Because of the advantages of heuristic and metaheuristic algorithms, the usage of these techniques in industries is enhancing [1]. Figure 1 shows the domains of metaheuristic algorithms applications in different fields.

The majority of the metaheuristics have been expanded before the year 2000. These algorithms are named "classical metaheuristic algorithms" in this survey. The aforementioned classical algorithms are genetic algorithms (GA) [2], particle swarm optimization (PSO) [3], ant colony optimization (ACO) [4], genetic programming (GP) [5], differential evolution (DE) [6], simulated annealing (SA) [7], tabu search (TS) [8], greedy randomized adaptive search procedure (GRASP) [9], artificial immune algorithm (AIA) [10], iterated local search (ILS) [11], chaos optimization method (COM) [12], scatter search (SS) [13], shuffled frogleaping algorithm (SFLA) [14], and variable neighborhood search (VNS) [15].

The new algorithms are named "new generation metaheuristic algorithms" that they are such as artificial bee colony $(A B C)[16]$, bacterial foraging (BFO) [17], bat algorithm (BA) [18], biogeography-based optimization (BFO) [19], cuckoo search (CS) [20], firefly algorithm (FA) [21], gravitational search algorithm (GSA) [22], grey wolf optimizer algorithm (GWO) [23], krill herd (KH) [24], social spider optimization (SSO) [25], symbiotic organisms search (SOS) [26], and whale optimization algorithm (WOA) [27].

Table 1 A review of a number of models presented in recent years

\begin{tabular}{|c|c|c|c|c|}
\hline Researcher & Solution method & Study origin & $U^{\mathrm{a}}$ & References \\
\hline Dagdelen and Johnson & Lagrangian relaxation method & General & & {$[43]$} \\
\hline Dowd & Geostatistical simulation & Gold deposit & $\checkmark$ & {$[44]$} \\
\hline Denby and Schofield & Genetic algorithm & General & $\checkmark$ & {$[45]$} \\
\hline Godoy and Dimitrakopoulos & Simulated annealing algorithm & A large gold mine, Australia & $\checkmark$ & {$[46]$} \\
\hline Gholamnejad et al. & Chance constrained programming & Gold mine & $\checkmark$ & {$[47]$} \\
\hline Lamghari and Dimitrakopoulos & Tabu search & $\begin{array}{l}\text { Two real-life datasets (copper and gold } \\
\text { deposits) }\end{array}$ & $\checkmark$ & [48] \\
\hline Sattarvand and Niemann-Delius & Ant colony optimization & A hypothetical iron ore deposit & & [49] \\
\hline Dimitrakopoulos and Jewbali & Stochastic integer programming & A large operating gold mine, Australia & & {$[50]$} \\
\hline Goodfellow and Dimitrakopoulos & Simulated annealing algorithm & $\begin{array}{l}\text { The BHP Billiton's Escondida Norte mine, } \\
\text { Chile }\end{array}$ & $\checkmark$ & {$[51]$} \\
\hline Asad et al. & $\begin{array}{l}\text { Stochastic network flow, Lagrangian } \\
\text { relaxation method }\end{array}$ & The copper mine & $\checkmark$ & {$[52]$} \\
\hline Lamghari et al. & Variable neighborhood descent algorithm & $\begin{array}{l}\text { Three different datasets (copper and gold } \\
\text { deposits) }\end{array}$ & $\checkmark$ & {$[53]$} \\
\hline Moosavi et al. & $\begin{array}{l}\text { Lagrangian relaxation method, genetic } \\
\text { algorithm }\end{array}$ & The Chadormalu iron ore mine, Iran & & {$[54]$} \\
\hline Lamghari et al. & Variable neighborhood descent algorithm & $\begin{array}{l}\text { Copper and gold deposits and MineLib } \\
\text { instances }\end{array}$ & & {$[55]$} \\
\hline Mokhtarian and Sattarvand & Imperialist competitive algorithm & A hypothetical copper deposit & & {$[56]$} \\
\hline Goodfellow and Dimitrakopoulos & $\begin{array}{l}\text { Simulated annealing algorithm, particle } \\
\text { swarm optimization, differential evolu- } \\
\text { tion }\end{array}$ & $\begin{array}{l}\text { The real-world copper-gold mining } \\
\text { complex }\end{array}$ & $\checkmark$ & {$[57]$} \\
\hline Khan & $\begin{array}{l}\text { Particle swarm optimization, bat algo- } \\
\text { rithm }\end{array}$ & Copper deposit & $\checkmark$ & {$[58]$} \\
\hline Alipour et al. & $\begin{array}{l}\text { Robust counterpart linear optimization, } \\
\text { genetic algorithm }\end{array}$ & A hypothetical copper mine & $\checkmark$ & {$[59]$} \\
\hline Dimitrakopoulos and Senécal & Multi-neighborhood tabu search & $\begin{array}{l}\text { Two case study (copper and gold depos- } \\
\text { its) }\end{array}$ & $\checkmark$ & {$[60]$} \\
\hline Chatterjee and Dimitrakopoulos & $\begin{array}{l}\text { Lagrangian relaxation method, subgradi- } \\
\text { ent method, branch and cut algorithm }\end{array}$ & Copper deposit & $\checkmark$ & {$[61]$} \\
\hline
\end{tabular}

auncertainty-based model 
Fig. 1 The share of metaheuristic algorithms application in different fields (in percentage)

Fig. 2 The share of related studies about optimization for classical metaheuristics

Fig. 3 The share of related studies about optimization for new generation metaheuristics

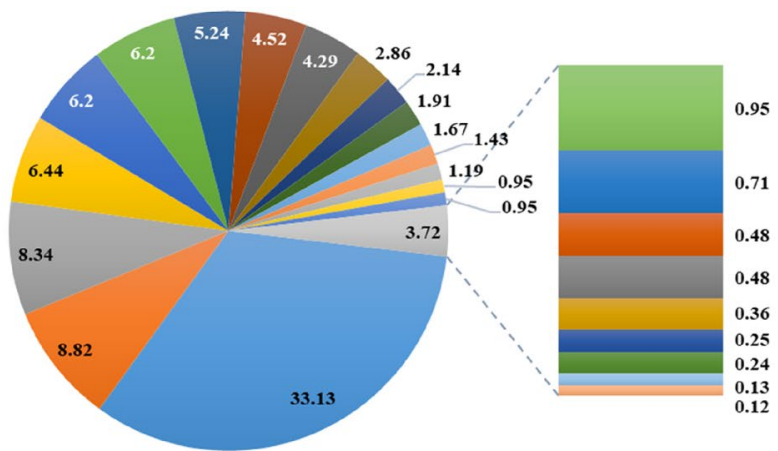

- Numerical Problems

a Scheduling

- Transportation

- ICT

ail and Energy

anriculture

- Water Management

a Civil Aviation

$\backsim$ Military and Defence
Data Mining

a Combinatorial Optimization Problems

angineering Design Problems

- Business, Finance, Economics

घmergency and Disaster Management

Biology

n Chemical Process Engineering

= Mining

- Music
- Electrical and Electronics

घ Communications

n Civil Engineering

n Image Processing

틀 Control Engineering

Medical

- Manufacturing and Production

n Traffic Control
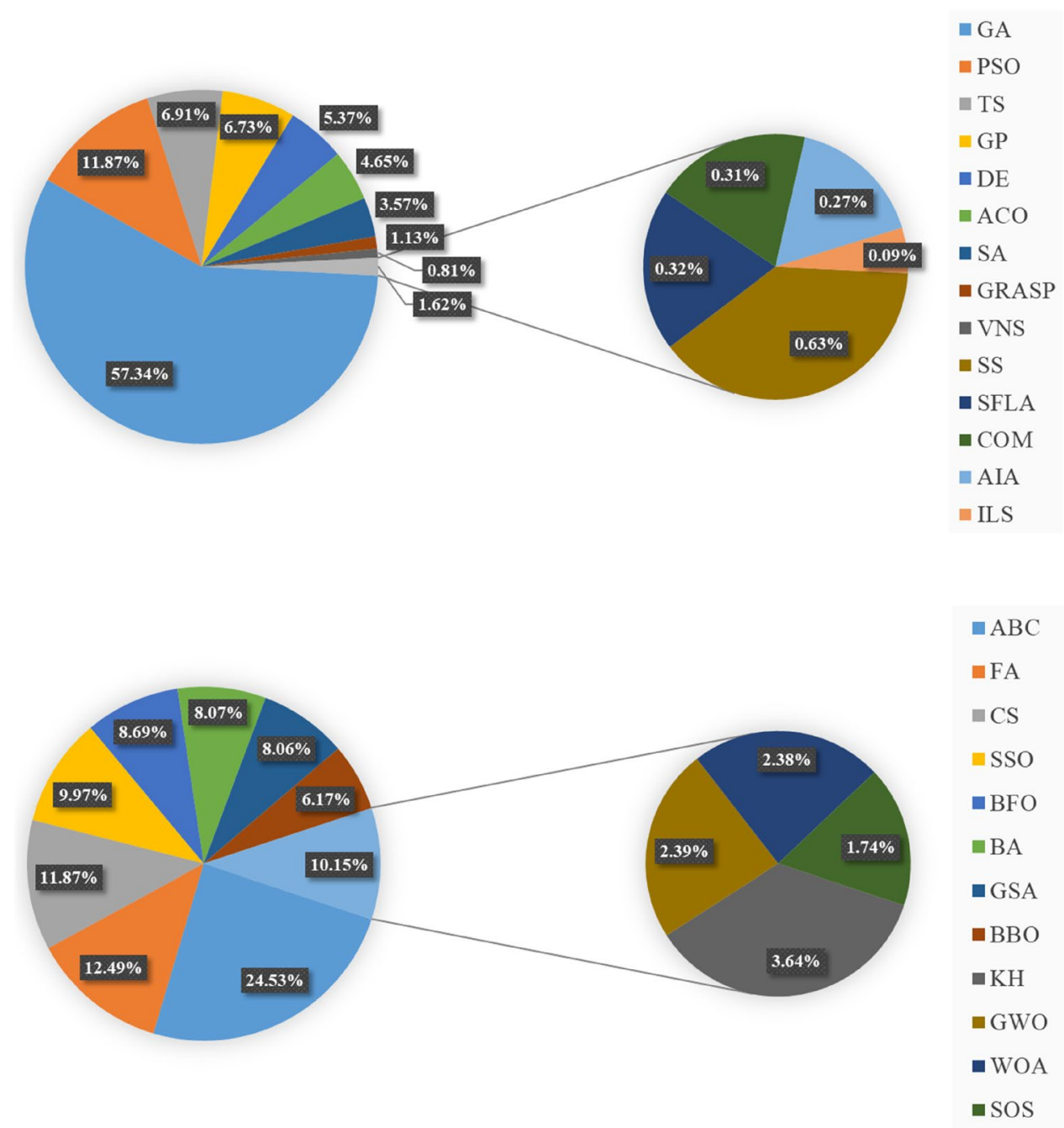

SN Applied Sciences 
The percentage of related studies about optimization for the classical and new generation metaheuristics is presented in Figs. 2 and 3.

In this study, the applications of metaheuristic algorithms have been dissected to solve the LTPS problem in open-pit mines, taking into account uncertainty. Using the hybrid technique by augmented Lagrangian relaxation (ALR) and GWO, the LTPS problem has been elucidated under grade uncertainty condition. Here, the ALR technique is applied to the LTPS problem to improve its performance and thus to speed up the convergence. Likewise, the GWO technique is applied thereon to update the Lagrangian multipliers. The proposed model has been compared with the results of the hybrid methods gained from the classic Lagrangian relaxation (LR) and ALR methods integrated with the BA, PSO, GA, the traditional subgradient method (SG), and conventional method without using the Lagrangian approach (Conv.). The case study with its constraints is evaluated to inspect the efficacy of the proposed method. Actually, the proposed method can be solved LTPS problems in rational time with a highquality solution.

The subsequent part of this paper is scheduled as below. Sections 2 and 3 indicate the objective functions and their associated constraints. Also, these sections indicate a summary of the methodology and the proposed model will be developed. Section 4 includes an evaluation of the outcomes. The established models are now validated. In Sect. 5, the results are analyzed and discussed. Finally, Sect. 6 shows the deduction.

\section{The long-term production scheduling problem formulation under grade uncertainty}

During several years, the LTPS has been implemented to assess production purposes and material flow in the ore [28]. It is typically simply represented and developed as a linear problem. To take these decision-making targets, the most straightforward way is to develop a full-space optimization model. Here, in each time period of the scheduling horizon, the availability constraints are incorporated into the model. Equation (1) provides the objective function (OF) of the LTPS problem and Eqs. (2)-(7) display the model constraints.

Maximize $Z=\sum_{n=1}^{N} \sum_{t}^{T} \frac{N V_{n}^{t}}{(1+r)^{t}} \times X_{n}^{t}$

$\sum_{n=1}^{N}\left(g_{n}-G_{\max }\right) \times O_{n} \times X_{n}^{t} \leq 0$

$$
\sum_{n=1}^{N}\left(g_{n}-G_{\min }\right) \times O_{n} \times X_{n}^{t} \geq 0
$$

$$
\sum_{t=1}^{T} X_{n}^{t} \leq 1, \quad \forall n=1,2,3, \ldots, N
$$

$\sum_{n=1}^{N}\left(O_{n} \times X_{n}^{t}\right) \leq P C_{\text {max }}$

$$
\sum_{n=1}^{N}\left(O_{n}+W_{n}\right) \times X_{n}^{t} \leq M C_{\max }
$$

$$
\begin{aligned}
& Y X_{k}^{t}-\sum_{y=1}^{l} \sum_{r=1}^{t} X_{y}^{r} \leq 0, \forall y=1,2, \ldots, l, \forall k=1,2, \ldots, N, \\
& \forall t=1,2, \ldots, T .
\end{aligned}
$$

The following indications were approved in the model constructed:

- $n$ : is the block identification number, $n=1,2, \ldots, N$;

- $N$ is the total number of blocks requiring to be scheduled;

- $t$ is the index of periods in scheduling horizon, $t=1,2$, $\ldots, T$;

- $T$ is the total number of scheduling periods;

- $\mathrm{NV}_{n}^{t}$ is the net value to be generated if block $n$ is mined in period $t$;

- $r$ is the discount rate in each period;

- $X_{n}^{t}$ is the binary variable;

- $G_{\max }$ is the value of the definite grade;

- $g_{n}$ is the mean grade of block $n$ and $O_{n}$ is the tonnage of ore in block $n$;

- $P C_{\max }$ is the processing capacity;

- $M C_{\max }$ is the mining capacity;

- $W_{n}$ is the tonnage of waste material in block $n$;

- $k$ is the index of a block regarded as extraction in period $t$;

- $Y$ is the total number of blocks that overly block $k$;

- $y$ is the counter for the $Y$-overlying blocks.

At present, the indicator kriging (IK) is one of the most widely used methods for grade estimation in mining projects. This technique was presented by Journel [29] to estimate the resources. The nature of the indicator method is binary data encoding depending on the cutoff value, $Z_{c}$. For the $Z(x)$ value, $i_{k}(x)=1$ if $Z(x) \geq Z_{C}$, and otherwise, $i_{k}(x)=0$. In fact, it is a nonlinear conversion of data value to binary system [30]. Outcome values 
between 0 and 1 for each estimation point provide a set of indicators-converted quantity using kriging, that can be expounded as the proportion of the block overhead the determined cutoff on data support and the probability that the grade is overhead the determined indicator [31]. In the optimization procedure of this paper, a mixed-integer programming (MIP) model is proposed to investigate grade uncertainty. In the block model, a possibility is allocated to each block $\left(U I_{n}\right)$, indicating the probability of $n$ for each block. Currently, the OF is organized such that earlier production periods are provided to mine higher-certainty blocks. Once extra information is available, uncertain blocks are generally postponed for later periods. Subsequently, another OF is introduced to the OF of the conventional model as follows:

Maximize $Z^{\prime}=\sum_{n=1}^{N} \sum_{t}^{T} \frac{N V_{n}^{t}}{(1+r)^{t}} \times U I_{n} \times X_{n}^{t}$.

This OF yields constraints (2)-(7).

\section{Structure of proposed model}

\subsection{Using the augmented Lagrangian relaxation
strategy for the reformulation of the long-term \\ 3.1 Using the augmented Lagrangian relaxation
strategy for the reformulation of the long-term production scheduling problem}

$$
\begin{aligned}
L(X, \lambda, \mu, v, \sigma)= & \operatorname{Min}-\sum_{n=1}^{N} \sum_{t}^{T} \frac{\mathrm{NV}_{n}^{t}}{(1+r)^{t}} \times \mathrm{UI}_{n} \times X_{n}^{t}+\frac{\sigma}{2} \times \sum_{t}^{T}\left(\left(X_{n}^{t}-1\right)+\frac{\lambda^{t}}{\sigma}\right)^{2} \\
& +\frac{\sigma}{2} \times \sum_{t}^{T}\left(\left(O_{n} \times X_{n}^{t}\right)-\mathrm{PC} \max +\frac{\mu^{t}}{\sigma}\right)^{2} \\
& +\frac{\sigma}{2} \times \sum_{t}^{T}\left(\left(O_{n}+W_{n}\right) \times X_{n}^{t}-M C_{\max }+\frac{v^{t}}{\sigma}\right)^{2}
\end{aligned}
$$

constant Lagrangian function is formulated by assigning non-negative Lagrangian multipliers $\lambda^{t}, \mu^{t}$ and $v^{t}$ with respect to processing type in period $t$ [37].

The ALR method has the potential to be used to solve the proposed problem. According to the function introduced by Andreani et al. [38], the ALR method is employed to generate a feasible solution for the original problem effectively. Given the reformulation above, once the coupling constraints (4)-(6) are relaxed, the resulting model (8) can be divided into several subproblems. The original (maximization) OF is on a par with the minimization of a revised OF. Here, the OF has been defined as follows:

Min $Z^{\prime \prime}(X)=-Z^{\prime}(X)$.

Relaxing constraints (4)-(6) yields the following ALR problem:

$$
\begin{aligned}
L(X, \lambda, \mu, v, \sigma)= & \operatorname{Min} Z^{\prime \prime}(X)+\frac{\sigma}{2} \times \sum_{t}^{T}\left(\left(X_{n}^{t}-1\right)+\frac{\lambda^{t}}{\sigma}\right)^{2} \\
& +\frac{\sigma}{2} \times \sum_{t}^{T}\left(\left(O_{n} \times X_{n}^{t}\right)-P C_{\max }+\frac{\mu^{t}}{\sigma}\right)^{2} \\
& +\frac{\sigma}{2} \times \sum_{t}^{T}\left(\left(O_{n}+W_{n}\right) \times X_{n}^{t}-M C_{\max }+\frac{v^{t}}{\sigma}\right)^{2} .
\end{aligned}
$$

According to the objective function, the formulation can be written as:
The LR method is regarded as a mathematical tool for MIPs. In introducing this method in LTPS, system constraints are relaxed and introduced to the OF by Lagrangian multipliers [32]. Afterward, the relaxed problem is decomposed into a further controllable subproblem for individual units and solved using dynamic programming [33]. Due to system constraint violations, the subgradient method is used to update multipliers [34]. The main idea of the LR method is system constraint relaxation by Lagrangian multipliers [35]. Then, the relaxed problem is decoupled into separate smaller subproblems [36]. The 


\subsection{Lagrangian multipliers updating with the grey wolf optimizer}

Given Lagrangian multipliers, they should be adjusted carefully to take advantage of the ALR. Most references to update Lagrangian multipliers utilize the subgradient method and some heuristic methods simultaneously to get a quick solution. Metaheuristic algorithms are considered suitable for solving the predicted problem. This study applies GWO to update Lagrangian multipliers and enhance ALR performance and compared it with the results of using the hybrid methods of $L R$ and ALR with the BA, PSO, GA, and SG.

In 2014, Mirjalili et al. [23] presented the GWO based on the social leadership of the grey wolves and their hunting group behavior in nature. As shown in Fig. 4, the group of the grey wolves is divided into alpha $(a)$, beta $(\beta)$, delta $(\delta)$, and omega $(\omega)$ in terms of social hierarchy behaviors [39]. Alpha is responsible for making major decisions. Beta, delta, and omega are in the lower ranks of dominance. In fact, according to this novel metaheuristic algorithm, the most appropriate solution considered to alpha and then assumed to beta and delta, respectively [40]. As well as, the remaining solutions belong to omega. The initial appropriate wolves that are closer to the prey are $(\alpha),(\beta)$ and $(\delta)$, which lead the remnant of the group $(\omega)$ to search for a prey in promising regions. Chasing, encircling, and attacking prey are the major hunting procedures of wolves [41]. The behavior of grey wolves in prey encircling is formulated as follows [42]:

$\vec{D}=\left|\vec{C} \cdot \overrightarrow{X_{P}}(t)-\vec{X}(t)\right|$

$\vec{X}(t+1)=\overrightarrow{X_{p}}(t)-\vec{A} \cdot \vec{D}$,

where $\vec{D}$ demonstrates the distance among the grey wolf and the prey, $t$ indicates the number of the current iteration, $\vec{X}$ shows the grey wolf position vector, and $\overrightarrow{X_{P}}$ is the prey position vector. As well as, $\vec{A}$ and $\vec{C}$ are coefficient vectors that computed as follows:

$\vec{A}=2 \vec{a} \cdot \overrightarrow{r_{1}}-\vec{a}$

$\vec{C}=2 \cdot \overrightarrow{r_{2}}$

where $\overrightarrow{r_{1}}, \overrightarrow{r_{2}}$ indicate random vectors in $[0,1]$, and the factor of $\vec{a}$ is linearly reduced from 2 to 0 over the period of iterations.

Hence, the three major solutions obtained are stored, and other search factors update their locations based on the location of ideal search factors. Their locations can be updated by GWO as follows:

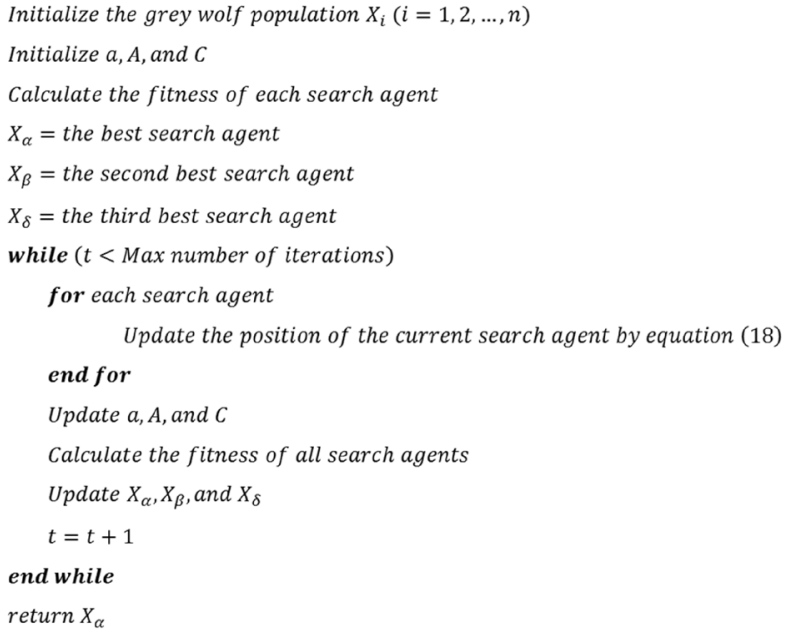

Fig. 5 Pseudocode of the GWO algorithm

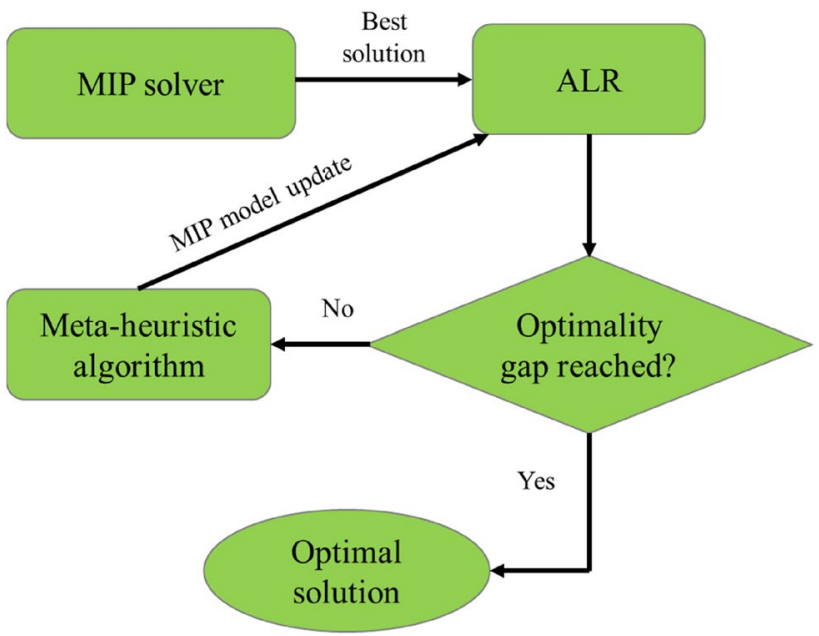

Fig. 6 Flowchart of the suggested model

$\overrightarrow{D_{\alpha}}=\left|\overrightarrow{C_{1}} \cdot \overrightarrow{X_{\alpha}}-\vec{X}\right|, \overrightarrow{D_{\beta}}=\left|\overrightarrow{C_{2}} \cdot \overrightarrow{X_{\beta}}-\vec{X}\right|, \overrightarrow{D_{\delta}}=\left|\overrightarrow{C_{3}} \cdot \overrightarrow{X_{\delta}}-\vec{X}\right|$

$\overrightarrow{X_{1}}=\overrightarrow{X_{\alpha}}-\overrightarrow{A_{1}} \cdot\left(\overrightarrow{D_{\alpha}}\right), \overrightarrow{X_{2}}=\overrightarrow{X_{\beta}}-\overrightarrow{A_{2}} \cdot\left(\overrightarrow{D_{\beta}}\right), \overrightarrow{X_{3}}=\overrightarrow{X_{\delta}}-\overrightarrow{A_{3}} \cdot\left(\overrightarrow{D_{\delta}}\right)$

$\vec{X}(t+1)=\frac{\overrightarrow{X_{1}}+\overrightarrow{X_{2}}+\overrightarrow{X_{3}}}{3}$.

The pseudocode of the GWO algorithm is demonstrated in Fig. 5. 
Table 2 Implementation of the proposed model for the synthetic dataset containing 4750 blocks

\begin{tabular}{lllll}
\hline Iteration & Method & Optimality gap & Method & Optimality gap \\
\hline 1 & LR-GWO & 0.088 & ALR-GWO & 0.056 \\
& LR-BA & 0.094 & ALR-BA & 0.062 \\
LR-PSO & 0.112 & ALR-PSO & 0.088 \\
LR-GA & 0.247 & ALR-GA & 0.123 \\
LR-SG & 2.07 & ALR-SG & 1.94 \\
& LR-GWO & 0.055 & ALR-GWO & 0.032 \\
& LR-BA & 0.069 & ALR-BA & 0.037 \\
LR-PSO & 0.098 & ALR-PSO & 0.049 \\
LR-GA & 0.163 & ALR-GA & 0.082 \\
LR-SG & 1.245 & ALR-SG & 0.664 \\
LR-GWO & 0.023 & ALR-GWO & 0.019 \\
LR-BA & 0.029 & ALR-BA & 0.021 \\
LR-PSO & 0.047 & ALR-PSO & 0.032 \\
LR-GA & 0.068 & ALR-GA & 0.045 \\
LR-SG & 0.621 & ALR-SG & 0.407 \\
\hline
\end{tabular}

Table 3 Parameters setting of metaheuristic methods

\begin{tabular}{lll}
\hline Algorithm & Parameters & Value \\
\hline GWO & $\vec{a}$ & $\begin{array}{l}\text { Linearly } \\
\text { decreased } \\
\text { from } 2 \text { to } 0\end{array}$ \\
& & 0.0 \\
BA & Minimum frequency $\left(f_{\text {min }}\right)$ & 2.0 \\
& Maximum frequency $\left(f_{\text {max }}\right)$ & 0.5 \\
& Loudness $(A)$ & 0.5 \\
& Pulse rate $(r)$ & 0.95 \\
& Constant $(a)$ & 0.05 \\
& Constant $(\gamma)$ & 0.4 \\
PSO & Minimum inertia weight $\left(W_{\max }\right)$ & 0.9 \\
& Maximum inertia weight $\left(W_{\min }\right)$ & 2 \\
& Personal learning coefficient $\left(c_{1}\right)$ & 2 \\
& Global learning coefficient $\left(c_{2}\right)$ & 0.8 \\
GA & Crossover percentage $\left(p_{\mathrm{c}}\right)$ & 0.1 \\
& Mutation percentage $\left(p_{\mathrm{m}}\right)$ & 0.03 \\
\hline
\end{tabular}

\subsection{The framework of the proposed model}

The hybrid method proposed in this paper entails taking two steps: (1) the Lagrangian function that updates Lagrange multipliers and (2) the constrained global maximization of the ALR function, where GWO is applied to find an optimal solution. The flowchart of the proposed method is shown in Fig. 6.
Table 4 Technical parameters

\begin{tabular}{ll}
\hline Parameters (unit) & Value \\
\hline Total block & 6854 \\
Block dimension $\left(\mathrm{m}^{3}\right)$ & $25 \times 25 \times 15$ \\
Discount rate $(\%)$ & 10 \\
Cutoff grade (\%) & 52 \\
Mining capacity (Mtone/year) & 25 \\
Processing capacity (Mtone/year) & 8.1 \\
Mining recovery (\%) & 90 \\
Processing recovery factor (\%) & 76 \\
Mine life (year) & 12 \\
\hline
\end{tabular}

Table 5 Number of model variables

\begin{tabular}{lr}
\hline Variables & Value \\
\hline Reserve constraints & 6854 \\
Iron ore grade constraints & 12 \\
Processing capacity constraints & 12 \\
Mining capacity constraints & 12 \\
Wall slope constraints & 82,248 \\
Binary constraints & 6854 \\
Total variables of model & 95,992 \\
\hline
\end{tabular}

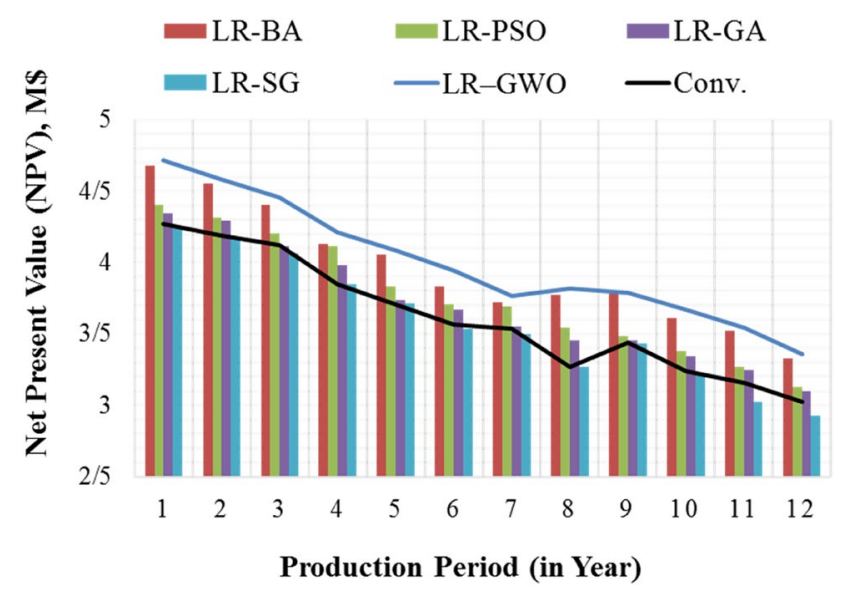

Fig. 7 Comparison of NPV obtained by LR-based presented methods considering grade uncertainty

\section{Numerical results}

This paper developed, applied, and tested the proposed model in MATLAB R2019a environment. The tests above have been conducted on a PC with Intel Quad Core, $3.5 \mathrm{GHz} \mathrm{CPU}$, and $32 \mathrm{~GB}$ of RAM. All formulations developed so far are verified by numerical experiments on a synthetic dataset with 4750 blocks. Table 2 implies 


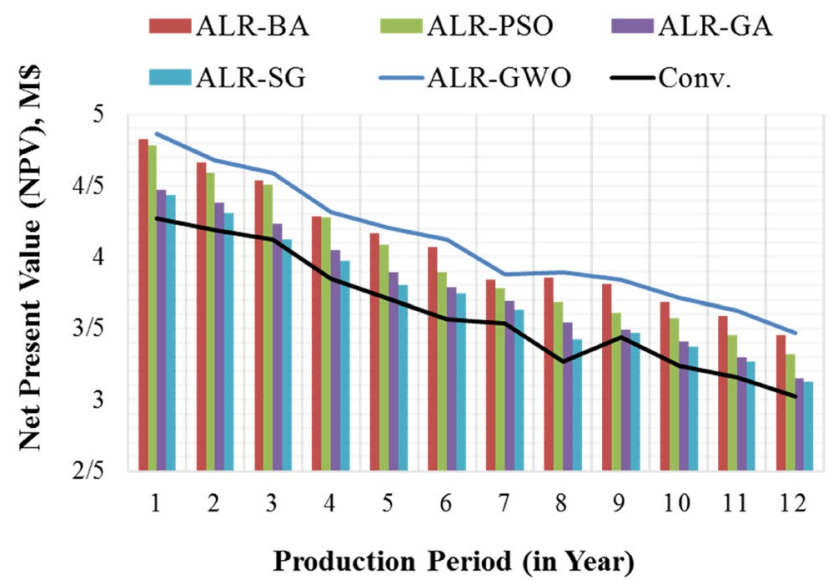

Fig. 8 Comparison of NPV obtained by ALR-based presented methods considering grade uncertainty

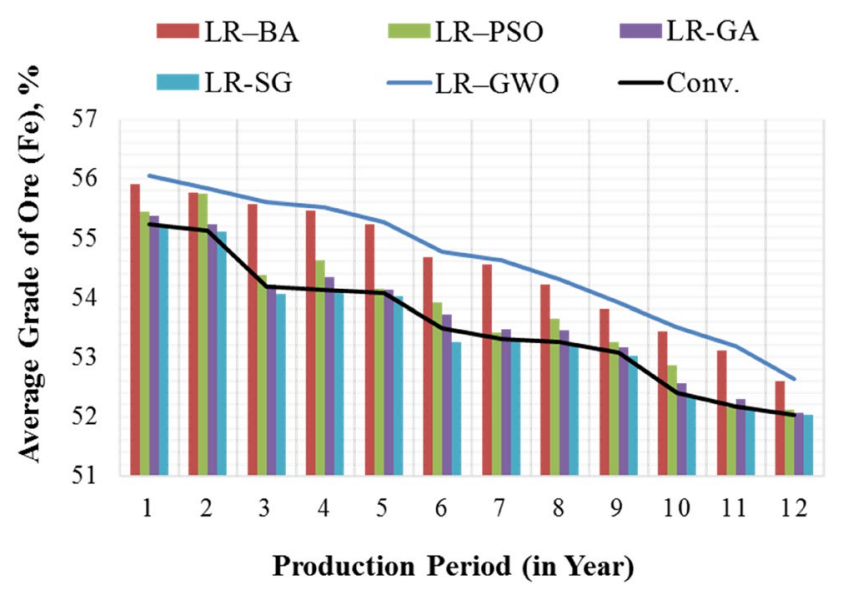

Fig. 9 Comparison of average ore grade obtained by LR-based methods considering grade uncertainty

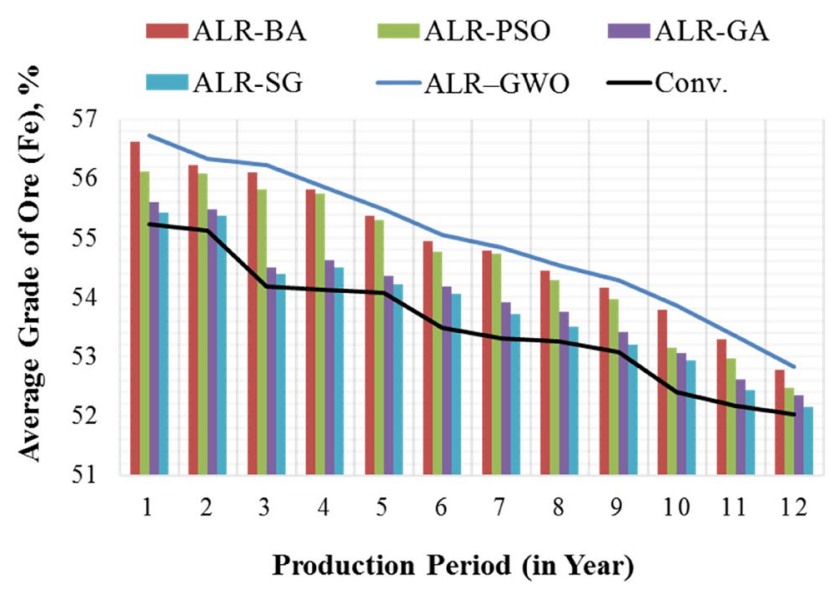

Fig. 10 Comparison of average ore grade obtained by ALR-based methods considering grade uncertainty

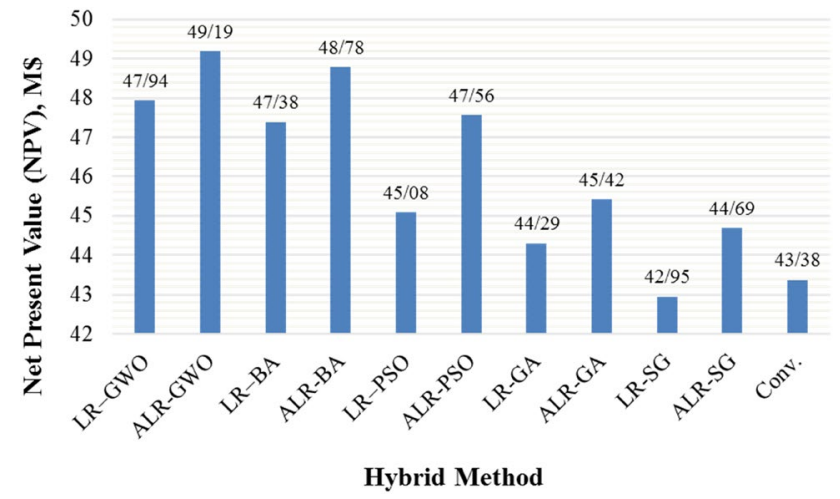

Fig. 11 Comparison of cumulative NPV in the total 12-year period obtained by hybrid methods

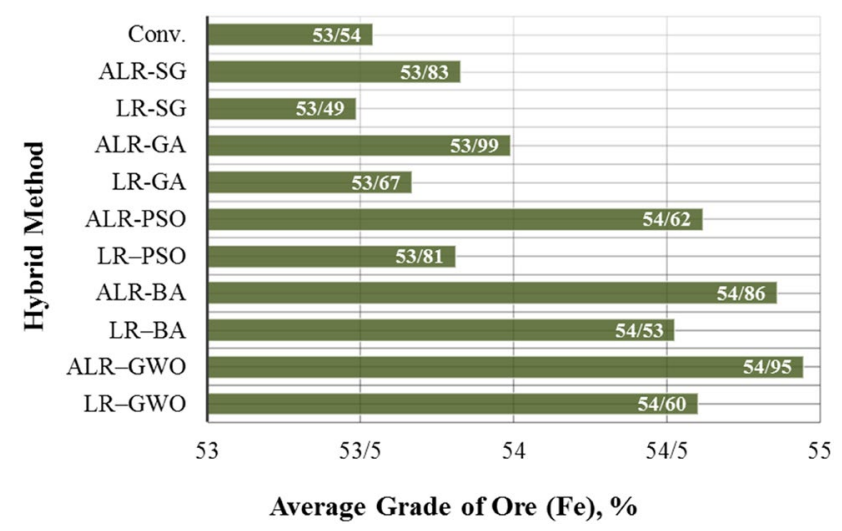

Fig. 12 Comparison of average ore grade in the total 12-year period obtained by hybrid methods

the outperformance of the ALR-GWO method in terms of the optimality gap. Also, the parameters were fixed according to the model changes and convergence. In this paper, various metaheuristic algorithms have been used including grey wolf optimizer algorithm, bat algorithm, particle swarm optimization, and genetic algorithm. Table 3 illustrates the parameters applied to metaheuristic methods. Furthermore, the maximum number of iterations was utilized as the criterion for terminating iterations set at 1000 following the observation of the convergence behavior of the algorithm.

The proposed integrated mine scheduling optimization framework has been applied to a real iron deposit. The iron deposit above contains 6854 blocks situated in Iran. Tables 4 and 5 provide the technical parameters and the number of model variables for the iron deposit, respectively.

The results of net present value (NPV) and average ore grade in uncertainty-based condition for the case study using LR-metaheuristic and ALR-metaheuristic approaches 
in the twelve production periods are illustrated in Figs. 7, 8,9 , and 10 . Besides, the outcomes of NPV and average ore grade obtained by the conventional method without using the Lagrangian relaxation approach are presented.

The comparison of cumulative NPV and average ore grade in the total period in uncertainty-based condition for the case study obtained by all of the solution approaches is demonstrated in Figs. 11 and 12. As outcomes are shown, the ALR-GWO method generates more appropriate results than the other presented methods. The cumulative NPV and the average ore grade in the 12-year period considering grade uncertainty using the ALR-GWO method are $13.39 \%$ and $2.63 \%$ more than the conventional method, respectively.

\section{Discussion}

In open-pit mines, the LTPS problem is a MIP problem and is considered as the class of NP-hard problems that has to be solved in a reasonably small time due to the operational requirements. LTPS problem still cannot be considered a well-solved problem. Lagrangian relaxation method is widely and efficiently applied to solve optimization problems with complex constraints since it was brought forward in the 1960s. It often exploits the special decomposable structure of the initial problem to deal with large-scale complex problems in various fields, such as manufacturing scheduling, supply chain optimization, and production scheduling problems. The major purpose of this technique is to relax the structured coupling constraints, and surcharge them as a penalty to the objective function with the presentation of Lagrangian multipliers. A dual problem is thus organized with the multipliers as its variables and can be decomposed into easier solving subproblems. A two-level optimization framework is applied to solve the dual problem. The decomposed subproblems are solved individually at a low level with much less computational efforts and the Lagrange multipliers are coordinated and updated at a high level based on the level of constraints violation. In this study, the augmented Lagrangian relaxation method is used. The major advantage of the augmented Lagrangian relaxation over the Lagrangian relaxation is that the former may get a practicable solution in instances wherever initial and dual solutions give a duality gap.

To specify the multiplier values based on the past calculation outcomes, the authors use the subgradient method that is usually employed. According to the zigzag phenomenon and small steps, the subgradient method may join gradually on large problems. As noted in the literature review of manuscripts, the use of this method has increased in recent years by combining metaheuristic

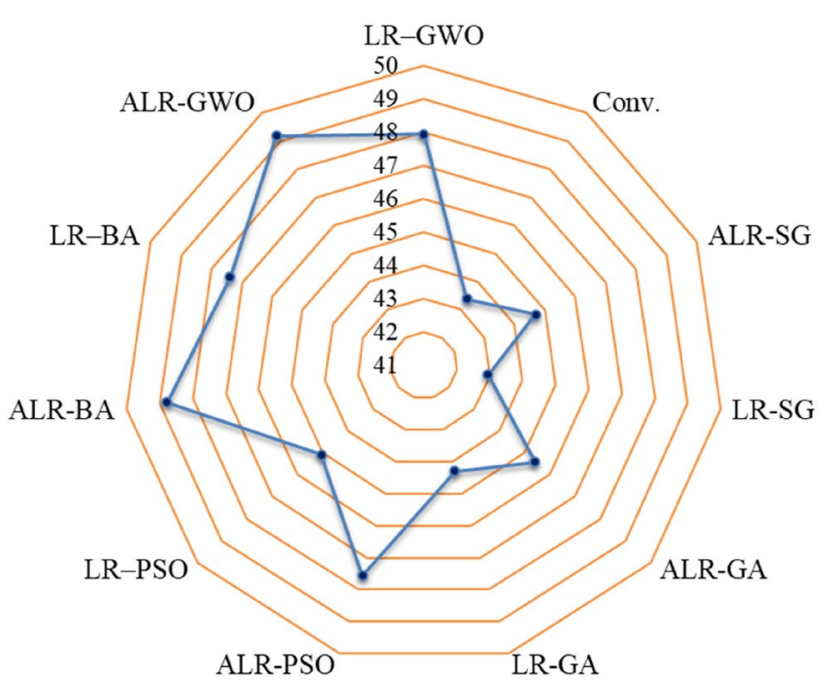

Fig. 13 General view of the cumulative net present value results

algorithms. They can be simply applied for the solution of hard optimization problems and they are responsible for great modeling flexibility. The results in different industries show the high performance of this model. In this research, different algorithms have been reviewed and tested for updating Lagrangian multipliers and chose the GWO algorithm based on the results of previous researches and the advantages of the various algorithms. According to the mentioned explanations, the combined method is a suitable option for the problem in the research and has the advantages such as ability to implement in large-scale long-term production scheduling problems in open-pit mines, reduce calculation time and cost, near-optimal final solution. Figure 13 provides an overview of the results of cumulative net present value over the entire 12-year period for the case study in terms of grade uncertainty. As can be seen in Fig. 13, ALR-GWO produces better result than other methods.

In fact, as stated, the greatest challenge in the LR method is to maximize the Lagrangian dual function efficiently, which is a concave multifaceted piecewise linear function. To determine multiplier values based on prior calculation results, the subgradient method has often been used. However, multipliers usually zigzag along the intersection of several facets of the Lagrangian dual function requiring a large number of repetitions to a maximum extent, albeit it may converge progressively. Based on the zigzag movement and small steps, the subgradient method may join step by step on big problems. Studying natural evolution influences metaheuristics global optimization algorithms. They can be utilized to solve hard optimization problems in charge of excellent modeling flexibility. In the context of the LR and ALR solutions to LTPS problem, metaheuristics approaches 
Table 6 An overview of the solution found by MATLAB for proposed models

\begin{tabular}{lllll}
\hline Methods & $\begin{array}{l}\text { Number of } \\
\text { blocks }(N)\end{array}$ & $\begin{array}{l}\text { Number of } \\
\text { periods }(T)\end{array}$ & $\begin{array}{l}\text { Optimality } \\
\text { gap }\end{array}$ & $\begin{array}{l}\text { CPU time } \\
\text { (min) }\end{array}$ \\
\hline ALR-GWO & 6854 & 12 & 0.043 & 25.19 \\
LR-GWO & 6854 & 12 & 0.049 & 26.08 \\
ALR-BA & 6854 & 12 & 0.051 & 27.39 \\
LR-BA & 6854 & 12 & 0.058 & 29.16 \\
ALR-PSO & 6854 & 12 & 0.057 & 31.26 \\
LR-PSO & 6854 & 12 & 0.066 & 33.55 \\
ALR-GA & 6854 & 12 & 0.071 & 34.14 \\
LR-GA & 6854 & 12 & 0.076 & 35.04 \\
ALR-SG & 6854 & 12 & 0.183 & 42.47 \\
LR-SG & 6854 & 12 & 0.255 & 44.51 \\
Conv. & 6854 & 12 & 2.138 & 236.49 \\
\hline
\end{tabular}

can be applied for dual variables. The main idea of the LR and ALR metaheuristics is that metaheuristics algorithms are integrated into the LR method to update the Lagrange multipliers. This method shows how LR is used in solving combinatorial optimization problems, including LTPS problem. According to the results of the averaging LR and ALR with GWO, BA, GA, and PSO algorithms, accelerating the convergence and greatly near-optimal solutions to LTPS problem can be finalized by the LR and ALR metaheuristics.

Table 6 shows the computation time and the optimality gap of each method. According to the evaluations, the CPU time is higher compared to other approaches that take advantage of the hybrid ALR-GWO technique proposed in this paper. Accordingly, these approaches drastically reduce computation time while keeping the optimality gap low. The latter implies the effectiveness of the proposed method. Besides, Fig. 14 displays that the iteration number according to the problem size of GWO is the minimum, and also, the iteration number of SG has high fluctuation when the problem scale gets bigger. Furthermore, the average cost-effectiveness ratio, best costeffectiveness ratio, worst cost-effectiveness ratio, and deviation are presented in Table 7. As shown in Table 7, the results of GWO are superior to the other methods studied in this paper.

\section{Conclusions}

This paper proposed a hybrid technique to solve the LTPS problem in open-pit mines since it is a large-scale, complex problem. A novel method was also introduced to optimize Lagrange multipliers in the hybrid ALR-GWO method and to compare it with the SG method in terms of performance. The result of the case indicated the effectiveness of the hybrid ALR-GWO method to find a feasible solution for

Table 7 Statistical results of the studied methods

\begin{tabular}{llllll}
\hline & GWO & BA & PSO & GA & SG \\
\hline $\begin{array}{l}\text { Average cost-effectiveness } \\
\text { ratio }\end{array}$ & 1.914 & 1.927 & 1.944 & 1.961 & 1.976 \\
$\begin{array}{l}\text { Best cost-effectiveness ratio } \\
\text { (min) }\end{array}$ & 1.752 & 1.769 & 1.788 & 1.807 & 1.822 \\
$\begin{array}{l}\text { Worst cost-effectiveness ratio } \\
\text { (max) }\end{array}$ & 1.937 & 1.932 & 1.924 & 1.912 & 1.908 \\
\begin{tabular}{l} 
Deviation (\%) \\
\hline
\end{tabular} & 0.383 & 0.367 & 0.351 & 0.332 & 0.318 \\
\hline
\end{tabular}

Fig. 14 Comparing proposed models in terms of iteration number

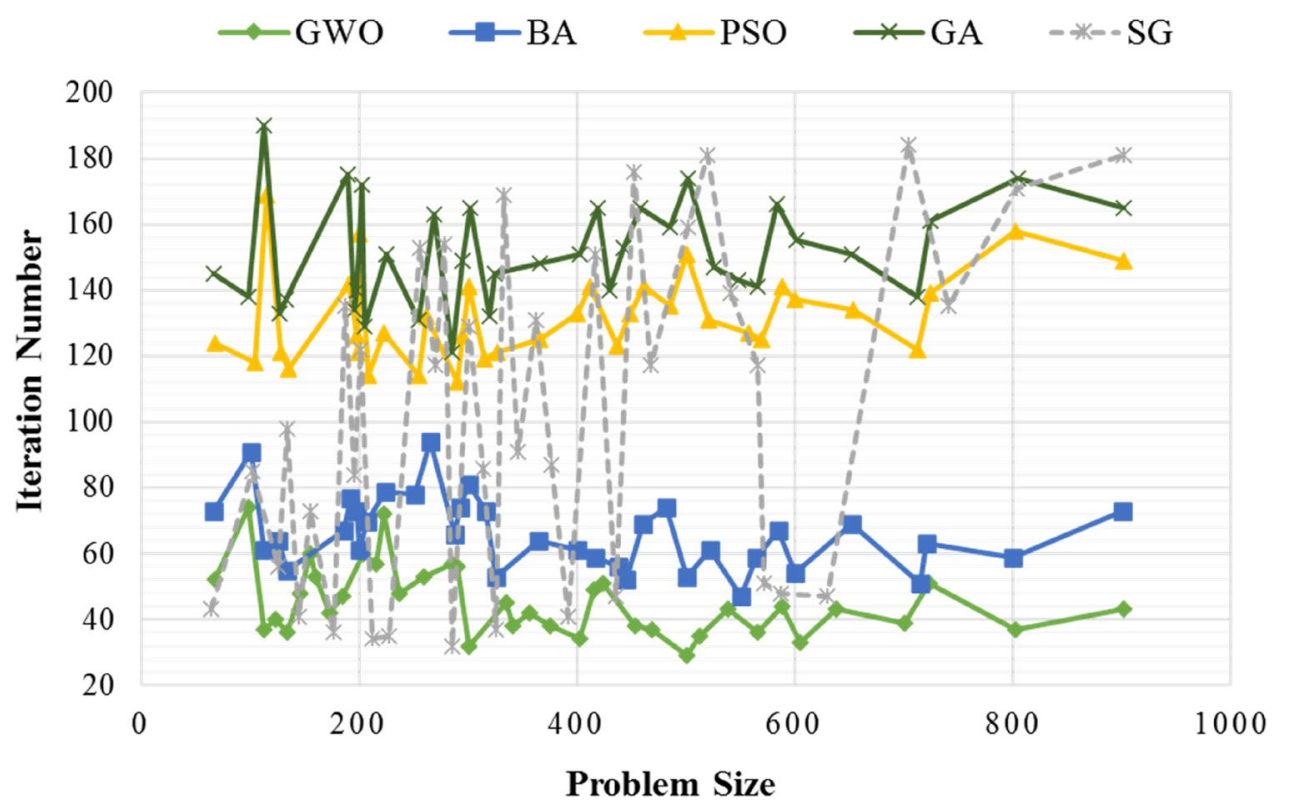


the LTPS problem. The hybrid strategy can produce a more effective solution to the extent of the near-optimal solution in comparison with the conventional method. Moreover, it was specified that the stable convergence property and prevention of early convergence are identified as the major advantages of the method suggested in this paper. In terms of cumulative NPV, average ore grade, and CPU time, results demonstrate that ALR-GWO generates best outcomes while satisfying constraints. The CPU time by the ALR-GWO hybrid method was $838.82 \%$ higher than the conventional method and also $8.73 \%, 24.09 \%, 35.53 \%$, $68.60 \%, 3.53 \%, 15.76 \%, 33.19 \%, 39.10 \%$, and $76.70 \%$, more than the ALR-BA, ALR-PSO, ALR-GA, ALR-SG, LR-GWO, LR-BA, LR-PSO, LR-GA, and LR-SG procedures, respectively.

According to the results, further studies are required such as the application of the model presented in this paper for other iron ore mines as well as mines with different minerals, also, use of other existed methods for minimizing of large-scale and complex models instead of the Lagrangian relaxation method, furthermore, implementing of other metaheuristic methods for the proposed model and comparing their results with the methods presented in this research, besides, utilization of different variants of GWO such as binary grey wolf optimizer (BGWO) and multi-objective grey wolf optimizer (MOGWO) for implementation on LTPS problems, and finally, examining the effect of other uncertainties separately or simultaneously for the long-term production scheduling in future research.

\section{Compliance with ethical standards}

Conflict of interest On behalf of all authors, the corresponding author states that there is no conflict of interest.

\section{References}

1. Turgut MS, Turgut OE (2020) Differential evolution based global best algorithm: an efficient optimizer for solving constrained and unconstrained optimization problems. SN Appl Sci 2:600

2. Goldberg DE (1989) Genetic algorithms in search. Optim Mach Learn

3. Wei Y, Qiqiang L (2004) Survey on particle swarm optimization algorithm. Eng Sci 5(5):87-94

4. Dorigo M, Birattari M (2010) Ant colony optimization. Springer, Berlin

5. Banzhaf W, Nordin P, Keller RE, Francone, FD (1998) Genetic programming: an introduction. 1,. Morgan Kaufmann, San Francisco

6. Storn R, Price K (1997) Differential evolution-a simple and efficient heuristic for global optimization over continuous spaces. J Glob Optim 11(4):341-359

7. Van Laarhoven PJ, Aarts EH (1987) Simulated annealing. In: Simulated annealing: theory and applications, Springer, Berlin, pp 7-15
8. Glover F, Laguna M (1998) Tabu search. In: Handbook of combinatorial optimization. Springer, Berlin, pp 2093-2229

9. Marques-Silva JP, Sakallah KA (1999) Grasp: a search algorithm for propositional satisfiability. IEEE Trans Comput 48(5):506-521

10. Dasgupta D (2012) Artificial immune systems and their applications. Springer, Berlin

11. Lourenco HR, Martin OC, Stutzle T (2003) Iterated local search In: Handbook of metaheuristics. Springer, Berlin, pp 320-353

12. Li B, Jiang W (1997) Chaos optimization method and its application. Contr Theory Appl 4:613-615

13. Marti R, Laguna M, Glover F (2006) Principles of scatter search. Eur J Oper Res 169(2):359-372

14. Eusuff MM, Lansey KE (2003) Optimization of water distribution network design using the shuffled frog leaping algorithm. J Water Resour Plan Manag 129(3):210-225

15. Mladenovic N, Hansen P (1997) Variable neighborhood search. Comput Oper Res 24(11):1097-1100

16. Karaboga D (2005) An idea based on honey bee swarm for numerical optimization. Tech rep-tr06, Erciyes University, Engineering Faculty, Computer

17. Das S, Biswas A, Dasgupta S, Abraham A (2009) Bacterial foraging optimization algorithm: theoretical foundations, analysis, and applications. In: Foundations of computational intelligence, 3. Springer, Berlin, pp 23-55

18. Yang X-S (2010) A new metaheuristic bat-inspired algorithm. In: Nature inspired cooperative strategies for optimization (NICSO 2010). Springer, Berlin, pp 65-74

19. Simon D (2008) Biogeography-based optimization. IEEE Trans Evol Comput 12(6):702-713

20. Yang X-S, Deb S (2009) Cuckoo search via levy flights. In: 2009 World congress on nature and biologically inspired computing (NaBIC). IEEE, pp 210-214

21. Yang X-S (2010) Firefly algorithm, stochastic test functions and design optimisation. arXiv preprint arXiv:1003.1409

22. Rashedi E, Nezamabadi-Pour H, Saryazdi S (2009) GSA: a gravitational search algorithm. Inform Sci 179(13):2232-2248

23. Mirjalili S, Mirjalili SM, Lewis A (2014) Grey-Wolf optimizer. Adv Eng Softw 69:45-61

24. Gandomi AH, Alavi AH (2012) Krill herd: a new bio-inspired optimization algorithm. Commun Nonlinear Sci Numer Simul 17(12):4831-4845

25. Cuevas E, Cienfuegos M, ZaldiVar D, Perez-Cisneros M (2013) A swarm optimization algorithm inspired in the behavior of the social-spider. Expert Syst Appl 40(16):6374-6384

26. Cheng M-Y, Prayogo D (2014) Symbiotic organisms search: a new metaheuristic optimization algorithm. Comput Struct 139:98-112

27. Mirjalili S, Lewis A (2016) The whale optimization algorithm. Adv Eng Softw 95:51-67

28. Caccetta L, Hill SP (2003) An application of branch and cut to open pit mine scheduling. J Glob Optim 27:349-365

29. Journel AG (1983) Non-parametric estimation of spatial distributions. Math Geol 15(3):445-468

30. Gholamnejad J, Osanloo M (2007) Incorporation of ore grade uncertainty into the push back design process. J S Afr I Min Metall 107(3):177-185

31. Gholamnejad J, Moosavi E (2012) A new mathematical programming model for long-term production scheduling considering geological uncertainty. J S Afr I Min Metall 112(2):77-81

32. Vemuri $S$, Lemonidis $L$ (1992) Fuel constrained unit commitment IEEE Trans Power Syst 7(1):410-415

33. Shiina T, Watanabe I (2004) Lagrangian relaxation method for price-based unit commitment problem. Eng Opti 36(6):705-719

34. Lawphongpanich S (2006) Dynamic slope scaling procedure and Lagrangian relaxation with subproblem approximation. J Glob Optim 35:121-130 
35. Pang X, Gao L, Pan Q, Tian W, Yu S (2017) A novel Lagrangian relaxation level approach for scheduling steelmaking-refiningcontinuous casting production. J Cent South Univ 24(2):467-477

36. Fisher ML (1981) The Lagrangian relaxation method for solving integer programming problems. Manag Sci 27(1):1-18

37. Yamada S, Takeda A (2018) Successive Lagrangian relaxation algorithm for nonconvex quadratic optimization. J Glob Optim 71:313-339

38. Andreani R, Birgin EG, Martinez JM, Schuverdt ML (2008) On augmented Lagrangian methods with general lower-level constraints. SIAM J Optim 18(4):1286-1309

39. Tikhamarine $Y$, Souag-Gamane D, Kisi O (2019) A new intelligent method for monthly streamflow prediction: hybrid wavelet support vector regression based on Grey-Wolf optimizer (WSVRGWO). Arab J Geosci 12:540

40. Gupta S, Deep K (2019) An efficient Grey-Wolf optimizer with opposition-based learning and chaotic local search for integer and mixed-integer optimization problems. Arab J Sci Eng 44:7277-7296

41. Gupta S, Deep K (2020) Optimal coordination of overcurrent relays using improved leadership-based Grey-Wolf optimizer. Arab J Sci Eng 45:2081-2091

42. Verma AR, Gupta B (2020) A novel approach adaptive filtering method for electromyogram signal using Gray-Wolf optimization algorithm. SN Appl Sci 2:16

43. Dagdelen K, Johnson TB (1986) Optimum open pit mine production scheduling by Lagrangian parametrization. In: Proceeding of the 19th international symposium on the application of computers and operations research in the mineral industry, Pennsylvania State University, University Park, Pennsylvania, 13, pp 127-142

44. Dowd PA (1994) Risk assessment in reserve estimation and open-pit planning. Trans Inst Min Metall (Sect A: Min Ind) 103:148-154

45. Denby B, Schofield D (1995) Inclusion of risk assessment in open-pit design and planning. Trans Inst Min Metall (Sect A Min Ind) 104:67-71

46. Godoy M, Dimitrakopoulos R (2004) Managing risk and waste mining in long-term production scheduling of open-pit mines. SME Trans 316:43-50

47. Gholamnejad J, Osanloo M, Karimi B (2006) A chance-constrained programming approach for open pit long-term production scheduling in stochastic environments. J S Afr I Min Metall 106:105-114

48. Lamghari A, Dimitrakopoulos R (2012) A diversified Tabu search approach for the open-pit mine production scheduling problem with metal uncertainty. Eur J Oper Res 222(3):642-652

49. Sattarvand J, Niemann-Delius C (2013) A new metaheuristic algorithm for long-term open pit production planning. Arch Min Sci 58(1):107-118
50. Dimitrakopoulos R, Jewbali A (2013) A joint stochastic optimization of short and long term mine production planning: method and application in a large operating gold mine. IMM Trans Min Technol 122(2):110-123

51. Goodfellow R, Dimitrakopoulos R (2013) Algorithmic integration of geological uncertainty in push back designs for complex multi-process open pit mines. Min Technol 122(2):67-77

52. Asad MWA, Dimitrakopoulos R, Eldert JV (2014) Stochastic production phase design for an open pit mining complex with multiple processing streams. Eng Optim 46(8):1139-1152

53. Lamghari A, Dimitrakopoulos R, Ferland AJ (2014) A variable neighbourhood descent algorithm for the open-pit mine production scheduling problem with metal uncertainty. J Oper Res Soc 65:1305-1314

54. Moosavi E, Gholamnejad J, Ataee-Pour M, Khorram E (2014) Improvement of Lagrangian relaxation performance for open pit mines constrained long-term production scheduling problem. J Cent South Univ 21:2848-2856

55. Lamghari A, Dimitrakopoulos R, Ferland JA (2015) A hybrid method based on linear programming and variable neighborhood descent for scheduling production in open-pit mines. J Glob Optim 63:555-582

56. Mokhtarian $M$, Sattarvand J (2016) An imperialist competitive algorithm for solving the production scheduling problem in open pit mine. Int J Min Geo-Eng 50(1):131-143

57. Goodfellow R, Dimitrakopoulos R (2016) Global optimization of open pit mining complexes with uncertainty. Appl Soft Comput 40:292-304

58. Khan A (2018) Long-term production scheduling of open pit mines using particle swarm and bat algorithms under grade uncertainty. J S Afr I Min Metall 118:361-368

59. Alipour A, Khodaiari AA, Jafari A, Tavakkoli-Moghaddam R (2018) Uncertain production scheduling optimization in openpit mines and its ellipsoidal robust counterpart. Int J Manag Sci Eng Manag 13(4):245-253

60. Dimitrakopoulos R, Senécal R (2019) Long-term mine production scheduling with multiple processing destinations under mineral supply uncertainty, based on multi-neighbourhood Tabu search. Int J Min Reclam Env

61. Chatterjee S, Dimitrakopoulos R (2020) Production scheduling under uncertainty of an open-pit mine using Lagrangian relaxation and branch-and-cut algorithm. Int J Min Reclam Env 34(5):343-361

Publisher's Note Springer Nature remains neutral with regard to jurisdictional claims in published maps and institutional affiliations. 